\title{
Facility for extreme ultraviolet reflectometry of lithography optics
}

\author{
C Tarrio, S Grantham and T B Lucatorto \\ Photon Physics Group, National Institute of Standards and Technology, Gaithersburg, \\ MD 20899, USA \\ E-mail: ctarrio@nist.gov
}

Published 7 February 2003

Online at stacks.iop.org/Met/40/S229

\begin{abstract}
One of the most demanding applications of optics and optical coatings is in extreme ultraviolet lithography. Optical substrates must be polished to an accuracy of less than a nanometre, both the substrate and multilayer coatings must be atomically smooth, and the coatings must have a period that is controlled to a few tens of picometres. The metrology must have even greater capabilities to assure that the strict tolerances are met. Our extreme ultraviolet reflectometry facility has recently been furnished with the addition of a sample chamber that is capable of scanning the entire surface of a mirror up to $35 \mathrm{~cm}$ in diameter. The capabilities of this chamber will be described, as will the performance of the monochromator. Finally a plan will be presented to increase the stability of our monochromator in order to meet the needs of future generations of lithography tools.
\end{abstract}

\section{Introduction}

In the next few years semiconductor lithography will progress to a point at which conventional lenses can no longer be used. A leading candidate to become the next-generation lithography uses extreme ultraviolet (EUV) radiation with a wavelength of $13.4 \mathrm{~nm}$ [1]. This wavelength was chosen because of the availability of high-efficiency MoSi multilayer mirrors, which have reflectivities as high as 70\% [2] and long-term stability. An alpha-class tool is currently operating, which consists of a source, a condensing system, a mask and a four-mirror projection system [3]. The largest item in this system is the first condenser, which consists of six segments that, when assembled, form a mirror $27 \mathrm{~cm}$ in diameter with a $25 \mathrm{~cm}$ radius of curvature. It is important to have the capability to measure such a mirror in its fully assembled form due to the expense of assembling and aligning it to the precision necessary for EUV lithography (EUVL). We have recently installed a large sample chamber capable of mapping the entire surface of a mirror up to $35 \mathrm{~cm}$ in diameter and $40 \mathrm{~kg}$ in mass.

In order to print features of $70 \mathrm{~nm}$ or smaller, the optics must be polished to extremely high tolerances. The figure, or macroscopic shape, of each mirror must be accurate to a nanometre or less to achieve the requisite image quality, and the finish, or microscopic roughness, must be atomically smooth in order for the multilayer coatings to have the highest specular reflectivity attainable. The polished substrates are analysed using interferometry, atomic force microscopy and other analytical techniques. The MoSi coatings are then deposited. A typical multilayer coating has 40 periods, each of which is about $7 \mathrm{~nm}$ thick, meaning that the total coating is $280 \mathrm{~nm}$ thick, and the possibility exists that a small thickness error can lead to a significant degradation of the figure. EUV reflectometry is used to verify not only that each mirror has the highest attainable reflectivity, but also that the multilayer deposition process adds no figure errors. (A deviation from the prescribed periodicity, which would indicate a deviation from the prescribed figure for the coated mirror, would lead to a shift in the wavelength of peak reflectivity.) A reflectivity map of the surface is made, with the peak reflectivity and wavelength centroid recorded for many points along the surface, and this is compared with the target profile. The requirements for the uncertainties of the reflectometry facility have been outlined by Gullikson et al [4]: wavelength precision, $0.01 \%$; wavelength uncertainty, $0.03 \%$; positional uncertainty, $0.04^{\circ}$ in angle and $0.5 \mathrm{~mm}$ in linear displacement; peak reflectance precision, $0.12 \%$ and uncertainty, $0.5 \%$. Precision refers to the ability to measure small changes in reflectivity or peak wavelength with $95 \%$ confidence.

Our entire set-up will be described, and measurements on large mirrors will be presented.

\section{Monochromator}

The NIST/DARPA EUV reflectometry facility consists of three parts: the synchrotron ultraviolet radiation facility (SURF III) 
[5] storage ring, which provides continuum EUV radiation; the monochromator; and the reflectometer sample chamber. The monochromator is described in detail in [6]. Briefly, a Ni-coated toroidal mirror collects $20 \mathrm{mrad}$ horizontally and the full vertical extent of the emission from SURF III, and focuses the radiation onto an entrance slit. The radiation is deflected and refocused by a second toroid and is incident on one of two plane of different gratings line spacing: a 600 lines $\mathrm{mm}^{-1}$ grating blazed for $20 \mathrm{~nm}$, which operates between 8 and $40 \mathrm{~nm}$, or a 1500 lines $\mathrm{mm}^{-1}$ grating blazed for $8 \mathrm{~nm}$, used from 3 to $14 \mathrm{~nm}$. Wavelength is scanned by a plane Au-coated mirror that rotates and translates, reflecting the selected wavelength to the exit slit, which is re-entrant within the large sample chamber.

A unique feature of our facility is the tunablity of the source. SURF III is a single-magnet electron storage ring with a radius of $83.7 \mathrm{~cm}$. It can be operated reliably at electron beam energies from 100 to $380 \mathrm{MeV}$. This allows us to adjust the peak output wavelength to minimize out-of-band radiation due to scattered and higher-order radiation. By using the tunability of the source with a combination of filters and detectors, we can determine the effects of out-of-band radiation on the reflectivity measurement.

\section{Sample chamber}

Samples are mounted on a $42 \mathrm{~cm}$ diameter platen with six-axis motion. Figure 1 shows a schematic diagram of the motion axes of the sample chamber. The entire assembly rotates about a horizontal axis to set the angle of incidence, $\theta$. The transverse angle $(\Psi)$ can also be varied between $-30^{\circ}$ and $30^{\circ}$ to keep the reflection in the vertical plane when measuring curved samples, or to correct for wedge angles in flat samples when necessary. There are three degrees of translation, $35 \mathrm{~cm}$ in the horizontal direction, $x, 10 \mathrm{~cm}$ vertical, $y$, and $5 \mathrm{~cm}$ of 'piston' or $z$ motion. These three motions are used to bring a specific portion of the sample surface to the beam spot. Finally, there is a continuous azimuthal rotation of the platen, $\phi$. The combination of $x$ and $\phi$ motion allows us to scan the entire surface of a mirror up to $35 \mathrm{~cm}$ in diameter.

There are also two detector axes. The primary or $2 \theta$-axis is coincident with the $\theta$-axis of the sample. The $2 \theta$-axis operates on an arc, which is approximately $25 \mathrm{~cm}$ in radius from the centre of rotation of the $\theta$-axis and allows for the use of a single detector to measure both reflected and transmitted light. There is also a second degree of freedom for the detector carriage that consists of motion along an arc on the detector arm,

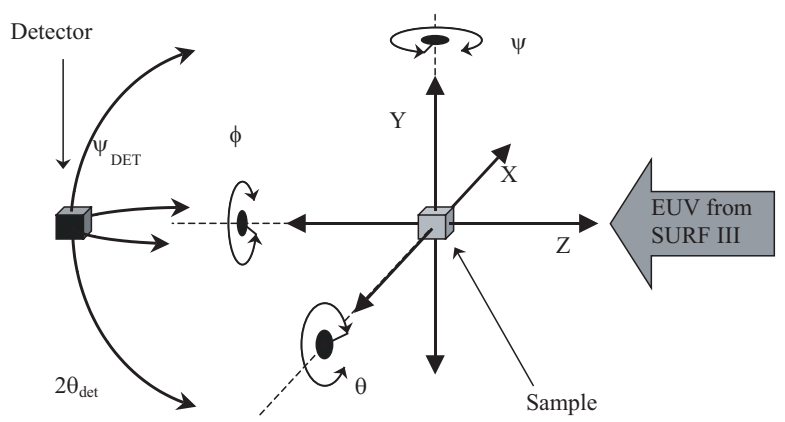

Figure 1. Schematic picture of the motion axes of the sample chamber. which is parallel to the $\theta$-axis. This motion serves two purposes: it allows us to switch detectors without breaking vacuum, and the additional $\pm 15^{\circ}$ of motion allows us to measure mirrors with slopes of up to $37.5^{\circ}$ by observing reflections out of the vertical plane. Given that our beam is $96 \%$ horizontally polarized, measurements with out-of-plane reflections must be corrected for the reduced reflectivity of reflections in the plane of polarization. This is a small correction, though, even at $15^{\circ}$.

This entire assembly is housed in a vacuum chamber that is $2 \mathrm{~m}$ in diameter and $3 \mathrm{~m}$ long and pumped by a dry roughing pump and two cryopumps. The chamber can be opened to the synchrotron $1-2 \mathrm{~h}$ after beginning the pumpdown cycle; this time may be reduced using available in-vacuum bakeout lamps. This is important in that it is a major factor when determining the sample turnaround time and the overall sample throughput of the instrument.

Alignment is done using an external laser that is reflected from a prism on the beamline's filter holder. The laser is aligned to the EUV beam by viewing both the visible and EUV at two points on the beam axis using phosphor screens. The position of the beam on the sample is viewed using an external video camera, while normal incidence is determined by retroreflecting through the exit slit and monitoring the signal with a photodiode. Figure 2 presents both vertical and horizontal scans, showing that we can determine normal incidence to $0.01^{\circ}$. The angle of incidence is then set using an angular encoder with resolution of $0.001^{\circ}$ located on the sample arm.

The reflected spot must be located accurately on the face of the $1 \mathrm{~cm}^{2}$ photodiode detector because the sensitivity of the detector varies across its face. This is done using a camera located inside the sample chamber that has a phosphor screen located $8^{\circ}$ from the photodiodes. The sample is swept through angle and the position of the reflected spot determined using the camera. The piston motion is adjusted until the position of the reflected spot does not change as the sample and detector are moved through a $\theta-2 \theta$ scan. The response of the detector over its central $80 \%$ is uniform to within $0.2 \%$, thus the uncertainty contribution of detector non-uniformity to our overall uncertainty is of the order of $0.01 \%$. The uniformity of the detector is measured periodically to ensure that no changes occur.

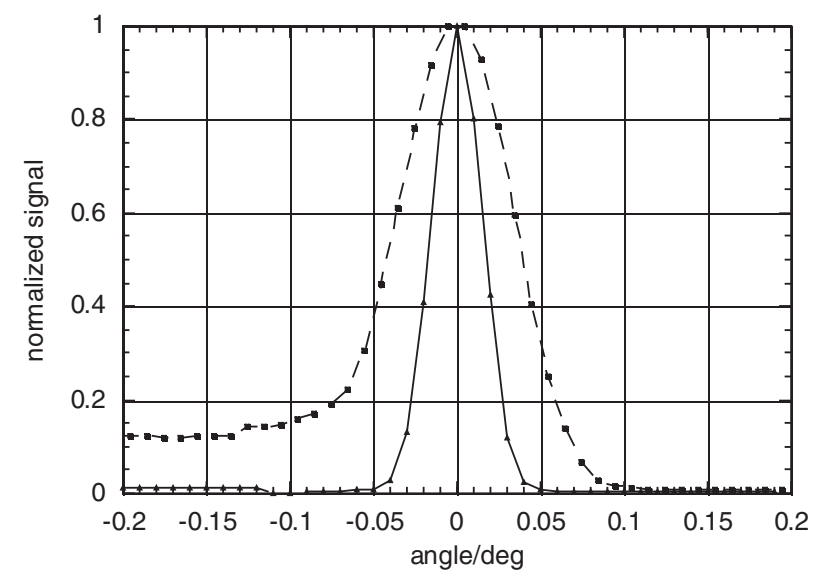

Figure 2. Vertical (__ ) and horizontal (- - - ) angle scans to determine normal incidence. 


\section{Measurement technique}

Measurements are made by first scanning wavelength with the detector intercepting the incoming beam and the sample parked out of the way. The sample and detector are then moved into the reflectivity measurement position. Because the number of electrons in the storage ring is constantly decaying, an accurate method must be used to normalize the signal. We have settled upon a unique method. A second exit slit, identical to the first in height and width, is located $2.5 \mathrm{~mm}$ from the real exit slit. A second EUV-sensitive photodiode is positioned behind this slit, measuring the beamline throughput shifted in wavelength by $0.25 \mathrm{~nm}$. Figure 3 shows a scan during which we measured the ratio of these two signals at $13.5 \mathrm{~nm}$ for a period of about $100 \mathrm{~min}$. The normalized signal has a best-fit slope of $0.01 \% \mathrm{~h}^{-1}$ and a standard deviation of $0.03 \%$.

In order to make accurate measurements, the spectral distribution of the incident beam must be well characterized so that any necessary corrections can be made to the data. We have used a unique method to make this characterization. As mentioned above, SURF III can be reliably operated over a wide range of stored beam energies, thus the output spectrum can be varied systematically. We have used this feature as well as filtering schemes to determine the amount of out-of-band radiation present in our beam. We have calculated the product of the output of SURF III, the transmission of the filters and the response of the photodiodes as a function of wavelength. Figure 4 shows three such combinations. As can be seen, a primary beam energy of $285 \mathrm{MeV}$ with a beryllium filter and $\mathrm{ZrSi}$-coated photodiode provides optimum measurement conditions. A beam energy of $380 \mathrm{MeV}$ with $\mathrm{C}$ filter with an unfiltered diode accentuates higher grating orders, while using $229 \mathrm{MeV}$, an unfiltered diode and a Be filter accentuates longwavelength scatter. We have made measurements of a single sample at four beam energies, with either $\mathrm{C}$ filter or Be filter, and filtered or unfiltered photodiode. Calculating the products as in figure 4 for these 16 combinations and the results for the reflectivity, we have used a least-squares procedure to determine the relative throughput of our monochromator for in-band, second-order, third-order and scattered radiation. Under optimum conditions there is no contribution from higher orders, and a $0.5 \%$ relative contribution from scatter. The total relative $(2 \sigma)$ uncertainty in the measurements is $0.3 \%$,

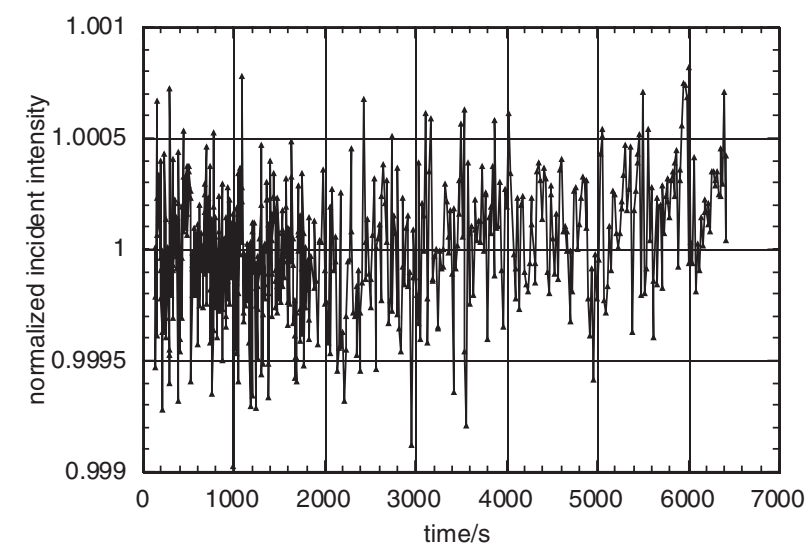

Figure 3. Normalization signal versus time. including a $0.25 \%$ uncertainty in determining the amount of scatter in the incident beam and $0.1 \%$ random measurement uncertainty.

Wavelength is calibrated by measuring the positions of filter absorption edges and the $13.59 \mathrm{~nm}$ absorption line in a $\mathrm{Kr}$ gas cell. There is a $0.01 \mathrm{~nm}$ uncertainty in our absolute wavelength scale. This is primarily due to thermal variations in the room where the monochromator is housed.

Figure 5 shows two measurements of a single flat mirror. These measurements were made five daysapart, and the sample

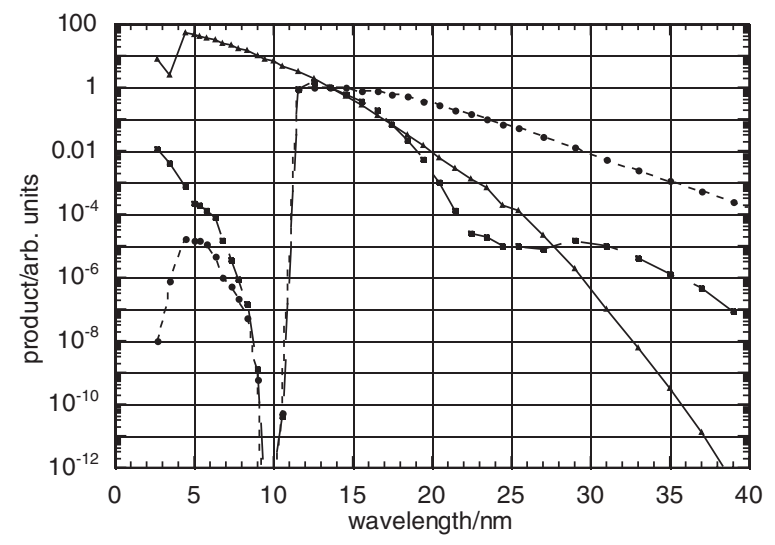

Figure 4. Product of synchrotron output, filter

transmission and photodiode response for: $285 \mathrm{MeV}$, Be filter, $\mathrm{Zr} / \mathrm{Si}$ coated photodiode $(-) ; 380 \mathrm{MeV}, \mathrm{C}$ filter, uncoated photodiode (- - - ); $229 \mathrm{MeV}$, Be filter, uncoated photodiode (- - - ).
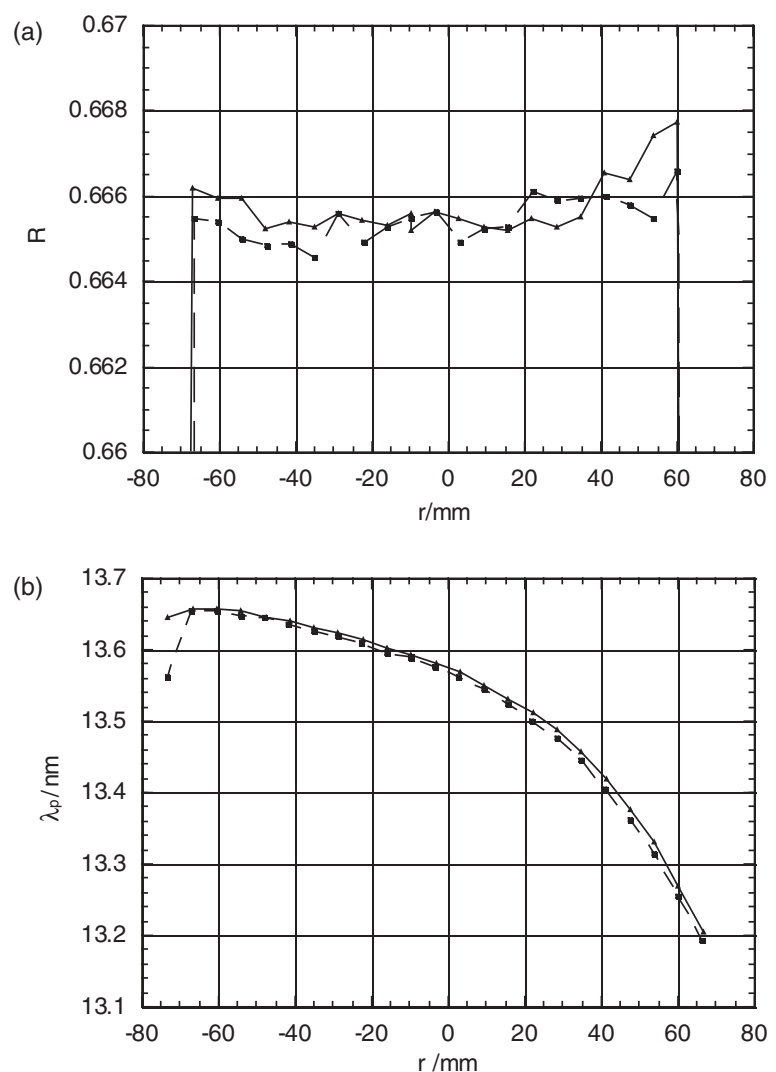

Figure 5. Two measurements of a single flat mirror made several days apart: $(a)$ peak reflectivity versus position; $(b)$ peak wavelength versus position. 
was unmounted and realigned between the measurements. The RMS differences point-by-point are $0.04 \%$ in reflectivity and $0.06 \%$ in wavelength. This repeatability meets the requirements for EUVL in reflectivity, but the wavelength stability must be improved by an order of magnitude.

\section{Summary and future directions}

We have demonstrated the capability to measure the reflectivity of mirrors used in EUV steppers. The sample chamber is capable of scanning the entire surface of mirrors with diameters up to $35 \mathrm{~cm}$ and mass up to $40 \mathrm{~kg}$. The accuracy achieved in reflectivity values is sufficient for characterization of production-generation stepper optics.

We have begun the design of a new housing for our monochromator. This design takes into account the $2^{\circ} \mathrm{C}$ temperature fluctuations encountered when the storage ring magnet and RF power supplies are energized. The new design will feature Invar supports for the collecting mirror and entrance slit. The scan mirror, which moves in two dimensions, will be replaced with a fixed mirror or two mirrors for different wavelength ranges, and a rotating grating will scan wavelength. The entire downstream assembly will be housed in a thermally controlled vessel.

\section{References}

[1] Stulen R H and Sweeney D W 1999 Extreme ultraviolet lithography Opt. Photon. News 35-8

[2] Bajt S, Alameda J, Barbee T W Jr, Clift W M, Folta J A, Kaufmann B and Spiller E 2001 Improved reflectance and stability of Mo/Si multilayers Soft X-ray and EUV Imaging Systems II: Proc. SPIE vol 4506, ed J A Folta and D A Tichenor, pp 65-75

[3] Tichenor D A et al 2001 Initial results from the EUV engineering test stand Soft $X$-ray and EUV Imaging Systems II: Proc. SPIE vol 4506, ed J A Folta and D A Tichenor, pp 9-18

[4] Gullikson E M, Mrowka S and Kaurmann B B 2001 Recent developments in EUV reflectometry at the advanced light source Proc. SPIE 4343 363-73

[5] Furst M L, Graves R M, Hamilton A, Hughey L R, Madden R P, Vest R E, Trzeciak W S, Bosch R A, Greenler L and Wahl P R D 1999 The conversion of SURF II to SURF III Proc. 1999 Particle Accelerator Conf. (New York) ed A Luccio and W Mackay (Piscataway, NJ: IEEE) pp 2388-90

[6] Callcott T A, O'Brien W L, Jia J J, Dong Q Y, Ederer D L, Watts R N and Mueller D R 1992 A simple variable-line-spacing monochromator for synchrotron light-source beamlines $\mathrm{Nucl}$. Instrum. Methods A 319 128-34 